\title{
EINLEITUNG
}

Gegenstand der Bibliographie sind die seit 1947 in der Wüste Juda westlich und nordwestlich des Toten Meeres gemachten Funde, für deren wichtigsten Teil der Name Handschriften vom Toten Meer* (Dead Sea Scrolls - manuscrits/rouleaux de la Mer Morte - מגילות יםדהמלח), genauer auch "Handschriften aus der Wüste Juda" (manuscrits du désert de Juda - מגילומר [מדר יהודה), üblich geworden ist. Die Funde kommen aus vier (fünf) voneinander unabhängigen Bereichen und laufen vom 4. Jahrtausend v. Chr. bis ins 9. Jahrhundert n. Chr. Der größte Teil stammt aus Khirbet Qumrân und Umgebung. Die Bibliographie erfaßt so

1. Grabungen und Funde in Khirbet Qumran und benachbarten Hohlen, zwischen Kh. Qumrdn und 'Ain FeSkha und in "Ain FeSkha selbst (8./7. Jahrh. v. Chr.'; 3./2. Jahrh. v. Chr. - 2. Jahrh. n. Chr.),

2. Grabungen und Funde in Höhlen im Wadi Murabba'at (vom 4. Jahrt. v. Chr. $a b^{1}$; 2. Jahrh. n. Chr.),

3. Grabungen und Funde in Khirbet Mird (5.-9. Jahrh. n. Chr.),

4. Untersuchungen und Grabungen in der Buqtah (8./7. Jahrh. v. Chr.) und

5. Funde unbekannter Herkunft (1./2. Jahrh. n. Chr.) $)^{3}$.

\section{Dazu kommen}

6. Arbeiten über die Damaskusschrift",

7. Arbeiten zur Ortskunde (Gesteins-, Pflanzen-, Wetterkunde, Beschreibungen, Karten) und

8. in Ausnahmen Arbeiten zu Einzelfragen wie der C14-Datierung,

auch wenn sie die Funde selbst nicht berühren. Solche Veröffentlichungen tragen ein $\dagger$ vor der Nummer.

Das Schrifttum zu $1-5$ ist der Absicht nach im Rahmen der p. XIIf genannten Regeln bis zum 31. Dezember 1955 vollständig notiert. Wichtige Veröffentlichungen sind noch bis Mai 1956 eingearbeitet worden. Ein Nachtrag ergänzt den Stoff bis zum Herbst 1956.

1 Uber die alteren Funde im Wadi Murabba'at $\rightarrow$ R. DE VAvx, 1110, daneben G. L. Harding, 512; J. M. Allegro, 1399, 170 f (CRAibl 1953, 333 erwahnt eine unveroffentlichte Arbeit von DE CONTENson, Le matériel chalcolithique de Muraba'at, Mémoire pour l'AIBL). Zur voressenischen Besiedlung von Qumrân und Umgebung $\rightarrow R$. DE VAUX, 1516, 535-537. 575, Hinweise auch in DE VAUX, 1120, 567: J. L. Kelso, 590, 145; M. NotH, 1483, 111f. Zur Buqê'ah $\rightarrow 295.789$. 1428. 1475. 1483.

Für Lageskizzen $\rightarrow$ 1399. 233. 1124. 859. 360. 357. 624 a. 396 u. a.

- Bis 1946 sind nur Textausgaben und vollstandige Ubersetzungen aufgenommen. Für altere Literatur $\rightarrow$ 551 . 836. 861 . 891 . 902. - Die Damaskusschrift gehơrt zur Literatur der Qumrân-Sekte. Außer den beiden (unvollst.) Handschriften, die S. SchechtrR 1896/97 in der Kairoer Geniza fand (CD), wurden bisher nur Reste einer Handschrift aus $6 Q$ (in 360, Appendix [ $\rightarrow$ : DE VAUX, $\$ 360,625]$ und 1151 'Hobhle 4' genannt) veröffentlicht $(\rightarrow 1404)$. Aus $4 Q$ sind Stücke von 7 Handschriften nachgewiesen $(\rightarrow 74,61$ [MILIK]). 
So ausgewählt, zählt die Bibliographie 1556 selbständige Veröffentlichungen und 352 Rezensionen von 616 Verfassern in 22 Sprachen 1 . Um den Satz zu vereinfachen, stehen die Arbeiten nach lateinischer (1-1246. 1395 - 1538), griechischer (1247-1250) und hebräischer (1251-1394. 1539 -1556) Schrift getrennt, ebenso die Titel der Zeitschriftenliste.

Schriftgrade unterscheiden im lateinischen Teil fünf Klassen von Veröffentlichungen:

A) TEXTAUSGABEN ${ }^{2}$, WICHTIGE BEARBEITUNGEN, UBERSETZUNGEN,

b) Längere primäre Arbeiten,

c) Kürzere primäre Arbeiten,

d) Längere sekundăre Arbeiten,

e) Kürzere sekundare Avbeiten.

Für den hebräischen Teil standen nur zwei Schriftgrade zur Verfügung. Sie unterscheiden primäre und längere sekundäre Arbeiten von den übrigen ${ }^{3}$. Der kurze griechische Teil ist einheitlich gesetzt.

Weiter gelten folgende Regeln:

Ubersetzungen von selbst verzeichneten Arbeiten führen deren Nummer mit a

$360 \mathrm{a}$

$939 x$

$\S$

$\S 1 \S 2$

$\$ 231$

$\& 144$

$+212$

$+6$

$(\rightarrow 360$ a; Gegenbeispiel $\rightarrow 17$ ), Dubletten mit geăndertem Titel die Nummer der Erstveröffentlichung mit $x(\rightarrow 939 x$; Gegenbeispiel $\rightarrow$ 942).

Rezensionen werden aufgenommen, wenn sie die Funde berühren und entweder (\$1) lang und kritisch oder (\$2) kurz und kritisch bzw. lang und berichtend sind. Sie erscheinen nur beim rezensierten Werk $(\rightarrow 2)$; dessen Nummer wird mit $\S$ unter dem Namen des Rezensenten angezeigt ( $\rightarrow$ BARDTKE).

Diskussionen werden mit \& an die Seitenzablen des diskutierten Vortrags angehängt $(\rightarrow 334)$. Die Teilnehmer werden nicht genannt (Ausnahme $\rightarrow$ 225).

Gemeinschaftsarbeiten werden aufgespalten, wenn die Anteile der Verfasser unterscheidbar sind. + weist auf den Anteil des/der Mitverfasser(s). Untrennbares wird beim erstgenannten Verfasser verzeichnet und unter dem Namen des folgenden durch + und Nummer angezeigt. Analog werden behandelt unter dem Namen des Nachschreibenden veröffentlichte Vortragsnachschriften $(\rightarrow 619)$, Bearbeitungen $(\rightarrow 134)$, Veröffentlichungen mit Nachschriften Dritter $(\rightarrow 208)$ und Auszïge, in denen der Referent ein Stück der Texte übersetzt $(\rightarrow 30)$.

1 Afrikaans, Dänisch, Deutsch, Englisch, Finnisch, Französisch, Griechisch, Holländisch/Flämisch, Italienisch, Japanisch, Jiddisch, Lateinisch, Neuhebrăisch, Norwegisch, Polnisch, Portugiesisch, Russisch, Schwedisch, Serbokroatisch, Spanisch, Tschechisch, Ungarisch.

2 Endgültig veröffentlicht wurden bisher nur die Funde aus $1 Q(\rightarrow$ 231. 232. 1364. 85), bis auf die aramäische sog. *Lamech «-Rolle (1QIV), die erst Anfang 1956 geöffnet wurde, aber statt des hypothetischen Lamechbuches $(\rightarrow 1084$. 406) einen Genesismidrasch enthält $(\rightarrow$ 38. 1403). Uber Stand und Plan der Veröffentlichung des übrigen Stoffes $\rightarrow$ 1013. 74. - Konkordanzen gibt es für $1 Q S$ $(\rightarrow$ 557), für $1 \mathrm{QS}, 1 \mathrm{QpH}$ und $\mathrm{CD}(\rightarrow 1298)$, für die nichtbihlischen $1 \mathrm{Q}-T$ Texte außer $1 \mathrm{QS}, 1 \mathrm{QpH}, 1 \mathrm{QM}, 1 \mathrm{QH}, 1 \mathrm{QIV}(\rightarrow \mathbf{8 5})$ und für die Hauptbegriffe in $1 \mathrm{QM}$ u. a. $(\rightarrow 1338)$. Eine 1Q-Konkordanz von BARDTKE soll 1958 erscheinen.

3 Die Einordnungen sind aber, wie die Angaben des hebräischen Teils überhaupt, manchmal zweifelhaft, weil ich die meisten Veröffentlichungen nicht selbst gesehen habe. 
Hebrdische Arbeiten eines im lateinischen Teil vertretenen Verfassers werden unter seinem Namen mit $\mathrm{H}$ angezeigt ( $\rightarrow$ BIRNBAUM).

Fortsetzungsartikel werden zu einer Nummer zusammengezogen, auch wenn der Titel schwankt $(\rightarrow 337)$.

Erganzungen $(\rightarrow 731)$ und Berichtigungen $(\rightarrow 72)$ werden zur betroffenen Arbeit gesetzt.

Auszüge in allgemeinen Literaturberichten und Zeitschriftenschauen ${ }^{1}$ werden, wenn lohnend, mit \% bei der betroffenen Arbeit vermerkt (bei Büchern und Sonderdrucken mit \$2). Das gilt auch für sekundäre Zeitungsartikel und Mitteilungen in Chroniken u. a., wenn eine gedruckte Quelle erkennbar ist $(\rightarrow 1108.85)$. Unauflosbares wird in Klasse e geführt $(\rightarrow$ 126), laufende Berichterstattung desselben Verfassers meist zu einer Nummer zusammengezogen $(\rightarrow 117)$. $0 \mathrm{~V}$ kennzeichnet nachschriflliche Vortragsauszüge.

Nicht notiert werden im allgemeinen lautende Bibliographien und Zeitschriftenschaven inner- und auBerhalb von Zeitschriften ${ }^{2}$, Artikel in Tages- und Wochenzeitungen ${ }^{3}$. Erwdhnungen in Jahresberichten wissenschaftlicher Gesellschaften, Bibelïbersetzungen". Ungedruckte Vorträge und Dissertationen werden nur vermerkt, wenn lohnende Inhaltsangaben gedruckt sind $(\rightarrow 350)$.

Für Anordnung und Schreibung gilt:

Der Stoff ordnet sich innerhalb der drei Schriftgruppen nach dem Alphabet der Verfasser, beim Verfasser nach Klassen, innerhalb der Klasse chronologisch; Bücher stehen vor Artikeln ( $\rightarrow$ DuPONT-SOMMER).

$M c$ - und $O^{\prime}$ - stehen unter $M$ und $O$, de, del, de la, des, lo, van, van den, van der, von unter dem Anfangsbuchstaben des folgenden Wortes, spanische Doppelnamen unter dem ersten, ungezeichnete Arbeiten unter ANONYM.

Als Titel gilt die Form, die sich bei Büchern auf dem Titelblatt, bei Artikeln in der Überschrift findet.

$1 \rightarrow$ n. 2.

a Die vollstăndigste einschlăgige Bibliographie ist $m$. W. P. NoBers jahrlicher Index bibliographicus biblicus in $\mathrm{Bibl}$; daneben $\rightarrow$ IZSch und im deutschsprachigen Bereich 336. 1435. An Zeitschriften mit laufender Bibliographie habe ich benutzt: ArchOF, ETL, EstEcl, Ciencia y Fe (San Miguel, Argentinien; span.-portug. Sprachgebiet), RHE, ThLZ, Theologische Revue (Müster).

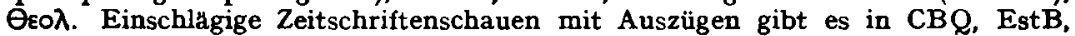
Sef (beide ab 1950), VD (Auswahl), ZAW, ohne Auszüge in JSemSt, NedThT, OLZ, RScPhTh, RThAM, ThZ, ZNW (unregelmäBig), רס"ק. Literaturberichte führen $\mathrm{HJ}$, Int, SvTKv (besonders für den skandinavischen Bereich). Einschlägige Literaturzeitungen sind BO, BL, OLZ, Scrin, ThLZ, Theologische Revue, 700 , auch ExpT. Die größten neueren Bibliographien zur Sache finden sich bei M. Burrows, 233/233 a und O. Eissfrldt, 1440; daneben $\rightarrow$ J. M. Allegro, 1399; J. van der Plozg, 1487. Ältere Bibliographien bieten (in der Reihenfolge des Erscheinens) 711. 572. 807. 109. 822. 75. 1298. 902. 735. 407. 569. 1124. 315. 755. 167. 934. 355. 1507; davon sind 902 (rd. 800 Titel) und $315 \mathrm{~m}$. W. die längsten.

s Ausgenommen sind israelitische Zeitungen und einige andere, die laufend über den Stand der Arbeit an den Funcen berichtet haben, wie Manchester Guardian, Le Monde, New York Times, Neue Zürcher Zeitung, Times u. a.

- Zur amerikanischen Revised Standard Version $\rightarrow$ 222. 798. 799. 1088. 1470, 78f. - Die Jerusalemer Bibel $\rightarrow$ 69. 1098) liegt jetzt mit gekürzten Anmerkungen auch in einem Band vor ( $\mathrm{La}$ Sainte Bible traduite en français sous la direction de l'École Biblique de Jérusalem, Paris 1956, XVI +1670, 9.4). 
Hebraische und japanische Arbeiten mit englischen Untertiteln behalten beide Die Schreibung der Namen Qumrân usw. schwankt und ist nicht vereinheitlicht. In der Groß- und Kleinschreibung und der Notierungsweise von Kapiteln und Versen kommen Abweichungen vor. Schriftgradwechsel werden nicht beachtet.

() [] Runde Klammern sind ursprünglich, eckige Zusatz.

Bei Büchern werden (Reihe), Erscheinungsort, Jahr, Seiten (ohne "S. " oder *p. ") genannt, bei periodischer Literatur die paginierende Einheit (Datum), Seiten.

(49)(49/50) Das Datum wird möglichst genau angegeben. 49 und $49 / 50(\rightarrow 11)$ bedeuten volle (12. 54) Jahre. Europaische Monate werden durch Ordnungszahlen bestimmt $(\rightarrow 6)$, he(.52) braische ausgeschrieben $(\rightarrow$ 1555). Ein bloßer Punkt vor der Jahreszahl $(\rightarrow 337)$ bezerchnet einen mir unbekannten Zeitpunkt oder Abschnitt des Jahres.

f Die Seitenzahl wird voll angemerkt. "f « bedeutet die folgende col. Seite. " col." weist auf Spaltenzählung.

Bei Arbeiten, die die Funde neben anderem behandeln, werden die einschlagigen

3-13:5f Seiten mit : hinter die Gesamtseitenzahl gesetzt $(\rightarrow 1)$. Bloßes : mit Seitenzahl ,$: 180 \mathrm{f}$ besagt, daB mir nur die einschlägigen Seiten bekannt sind $(\rightarrow 34)$, bloßes : nach 304-309: der Gesamtseitenzahl, daB sie mir nicht bekannt oder nicht erkennbar sind $(\rightarrow \$ 201)$. Bei hebräischen Zeitschriften mit englischen Zusammenfassungen ist zu beachten, daß die englischen Seiten, gewobnnlich römisch numeriert, meist in jedem Heft neu zahlen $(\rightarrow 1349)$.

Darüber hinaus gibt die Bibliographie gewisse Hinweise auf den TV xB Inhalt der verzeichneten Arbeiten. T führt Texte, V Úbersetzungen 1 QM* ein $(\rightarrow 6)^{1}$. * ("teilweise 《) kennzeichnet bloße Leseproben, häufige küızere Zitate $(\rightarrow$ 372) oder Stücke mir unbekannten Umfangs

T $731(\rightarrow 75)$. Bei mehrfach bearbeiteten Texten weist $T$ auf die erste/endguiltige Ausgabe $(\rightarrow 6)$. Abbildungen und Karten werden in einem $-/ 1 /-1-11$ Bildschlüssel $(\rightarrow$ p. VIII) erfaßt $(\rightarrow 4)$. Setzt sich eine Arbeit deutlich :1216 mit einer oder mehreren auseinander, wird die Gegenseite mit :

$\rightarrow: 1217$ und Nummer angeführt; umgekehrt weist $\rightarrow$ : auf Gegenveröffentlichungen hin $(\rightarrow 11)$. Bei ganz oder teilweise sekundären Arbeiten $<357$ a deutet $<$ mit Nummer die Quelle(n) an $(\rightarrow$ 83); $>$ und Nummer $>127$ zeigt, wo eine Arbeit sekundär benutzt wird $(\rightarrow 12)$. Andere Er[1 QIV] läuterungen stehen in eckigen Klammern $[\rightarrow 38]$.

Am SchluB sind die bisher veröffentlichten Texte und Ubersetzungen zusammengestelit ${ }^{2}$. Bloße Abbildungen werden kaum gebucht ${ }^{3}$. Die Identifizierungen gehen zu Lasten ihrer Urheber und sind manchmal fraglich, schon gar bei Vorveröffentlichungen. Um Platz zu sparen, habe ich die oft bearbeiteten Texte von den übrigen trennen müssen; über ihre Bedeutung sagt das nichts. Die Reihen-

1 Bekannte Werke werden nach Kapitel und Vers angeführt, $1 \mathrm{QH}$ und $1 \mathrm{QM}$ nach Kolumne [Seite in SUKRNIKs רצוא (1364/1364a)], Zeilen, alles übrige nach Kolumne, Zeilen.

Was auBerdem überhaupt da ist, ersieht man etwa aus $74(2-6 Q), 1516$ (7-10Q), 1110 (Mur), 1124, 28-33 (1-6Q. Mur, Mird, x), 1149 (1Q, 4Q, Mur, Mird, x). Unbelcannt ist noch, was $11 Q$ enthielt.

Einige davon arfa日t 735. - $\rightarrow$ 37. 84. 1215. 
folge ist hier wie dort: Kanonische Texte (einschließlich Phylakterien) - Kommentare - vor 1947 bekannte außerkanonische Texte - bisher unbekannte religiöse Texte - Urkunden, Briefe usw. Innerhalb der Gruppen folgen sich C, $Q$, Mur, Mird, $\mathbf{x}$, doch so, daß alle Handschriften desselben Werkes bei der erstaufgeführten notiert werden. - Regeln:

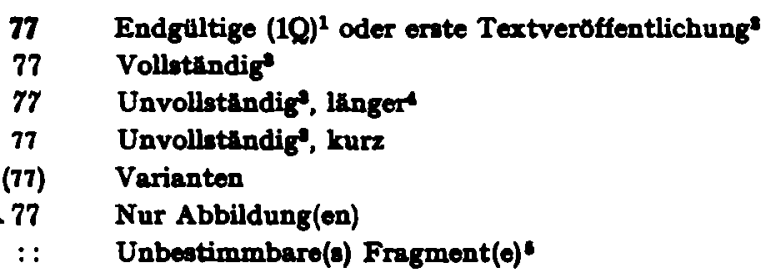

Es ist geplant, die Bibliographie in zwei oder drei Jahren in der ZAW selbst oder einem neuen Beiheft zu ergänzen und weiterzuführen.

$\rightarrow$ p. XII n. 2.

- Bei CD alle selbstandigen Ausgaben.

- Vollstandig; und unvollstandig; beziehen sich nur auf den (wo moglich, in der Spalte Inhalti genannten) veroffentlichten Umfang.

- 2 Spalton laufonden Texts und mehr.

- Bei vor 1947 bekannten Texten. 
